\title{
Editorial
}

Nephrology

Published online: September 5, 2018

\section{Have We Made "Rapid Progress" Understanding the Pathogenesis in Rapidly Progressive Glomerulonephritis?}

\author{
Nicholette D. Palmer ${ }^{\mathrm{a}}$ Lijun Ma ${ }^{\mathrm{b}}$ Barry I. Freedman ${ }^{\mathrm{b}}$ \\ ${ }^{a}$ Department of Biochemistry, Wake Forest School of Medicine, Winston-Salem, NC, USA; ${ }^{b}$ Department of Internal \\ Medicine, Section on Nephrology, Wake Forest School of Medicine, Winston-Salem, NC, USA
}

Many of us were drawn to the field of nephrology after experiencing the thrill of successfully treating an acutely ill patient with rapidly progressive glomerulonephritis (RPGN). The urgent need to recognize and institute aggressive treatment for these relatively rare cases remains of paramount importance, both to optimize patient survival and to reduce the likelihood of dialysis dependence. The characteristic crescentic renal histologic changes in RPGN can result from 3 different pathogenic mechanisms: deposition of immune-complexes, production of anti-glomerular basement membrane (GBM) antibodies or pauci-immune (vasculitic) processes that often include anti-neutrophil cytoplasmic antibodies (ANCA). Commonly used regimens to treat RPGN employ high-dose steroids and cytotoxic agents, with further protocols based on specific pathogenesis and extent and severity of organ involvement. This may include the use of plasmapheresis or plasma exchange to remove circulating anti-
() 2018 S. Karger AG, Basel

E-Mail karger@karger.com www.karger.com/ajn
GBM antibodies or treat ANCA-associated vasculitis when it presents with advanced nephropathy or pulmonary hemorrhage. However, no single or common biologic pathway has yet been identified across the 3 processes that produce crescentic glomerular disease and rapid loss of kidney function. This gap complicates the treatment of patients and limits our understanding of disease pathogenesis. RPGN being an uncommon disorder, appropriate biosamples to assist in identifying common versus independent pathways are frequently lacking.

In this issue of the American Journal of Nephrology, Wang et al. [1] performed weighted gene correlation network analysis (WGCNA) [2] aimed at identifying clusters (or modules) of highly correlated genes in patients with

Re:Wang Z, Chen X, Zhang D et al: Weighted Gene Co-Expression Network Analysis Reveals PYCARD Gene Plays a Key Role in Neutrophil Activation in Rapidly Progressive Glomerulonephritis. 
RPGN, summarizing these clusters using the module eigengene or an intra-modular hub gene, and relating modules to one another. Unlike other pathway development methods that are based solely on gene set enrichment analysis, WGCNA allows the identification of a putative hub gene that may play a key role in an index pathway or related pathways. Gene Ontology and Kyoto Encyclopedia of Genes and Genomes analysis were also performed to narrow down putative candidate pathways. Visualization of networks and screening of hub genes were performed using Cytoscape software. Multiple analysis modes increased the reliability of their results.

This study has strengths and weaknesses. An important strength is that the authors employed an unbiased approach to identify the most likely biological components involved in the pathogenesis of RPGN based on the global gene expression profile of a case/control subset from the dataset of glomerular transcriptome from European Renal cDNA Bank cases and living kidney donor controls (GeoData: GSE104948). The samples analyzed included cases with RPGN; however, review of the GSE10498 data revealed that all 22 cases had ANCA-associated vasculitis, none had anti-GBM disease or antigen-antibody deposition as the cause of RPGN. Hence, the top hit identified, the PYCARD gene, may not be surprising based on the known mechanisms in ANCA vasculitis. This was the first time that this gene was implicated in acute glomerulonephritis, and it supports the role of innate immunity in the development and progression of ANCA-associated vasculitis in RPGN.

This study was based only on in silico array data and lacked detailed clinical information; therefore, it is somewhat difficult to address the precise molecular mechanisms involved in RPGN. Additional insight could be gleaned from more comprehensive transcriptomic approaches, including RNA sequencing, which captures all transcripts as opposed to a targeted set. In addition, there were no protein-level analyses to support what the authors identified based on transcript data alone. Despite these limitations, it raises the possibility that either circulating or renal PYCARD could be a novel biomarker for RPGN because it is up-regulated by inflammation and apoptosis in human neutrophils [3]. It is currently unclear whether the PYCARD transcript information captured in GSE104948 was renal-specific or due to neutrophil infiltration involved in innate immunity. This will require further investigation on the circulating transcriptome of a well-characterized case/control cohort. The localization of PYCARD protein and mRNAs in human kidney sections may also help clarify these concerns. Regardless of these uncertainties, PYCARD is likely a novel biomarker for ANCA-associated RPGN. Animal models may also verify whether it plays a critical role in the development or progression of RPGN from causes other than ANCA.

PYCARD (PYD and CARD domain-containing protein, also known as apoptosis-associated speck-like protein containing a CARD) functions as a key mediator in apoptosis and inflammation. Through its role as an adaptor protein, PYCARD binds nucleotide-binding oligomerization domain-like receptors (NLRs) and recruits pro-caspase-1 to form an active inflammasome. Activated caspase- 1 can then cleave precursor cytokines to their biologically active forms, for example, interleukin- $1 \beta$ and interleukin-18, which induce synthesis of inflammatory and pro-fibrotic mediators [4]. Named by the scaffoldforming protein, the NLRP3 (NOD-like receptor family, pyrin containing domain-3) inflammasome has been implicated in kidney disease [5]. Specific to autoimmunerelated renal disease and as a result of caspase 1-dependent cytokines, autoreactive CD4+ T cell differentiation to Th1 and Th17 cells are important in the development of autoimmune disease [6]. Of note, the importance of inflammasome-derived pro-inflammatory cytokine production in crescentic glomerulonephritis has proven inconsistent in animal models [7].

In conclusion, this novel kidney tissue-based WGCNA performed in patients with RPGN identified a new pathway that may contribute to the development and/or progression of crescentic glomerulonephritis. Involved in inflammation, the PYCARD gene pathway has already been implicated in autoimmunity and autoimmune disorders. However, it is possible, perhaps likely, that this pathway plays a primary role in ANCA-associated RPGN since all cases had this disorder. Whether PYCARD plays a role in anti-GBM disease and antigen-antibody deposition associated forms of RPGN remains to be determined. Nonetheless, inhibiting the PYCARD pathway may provide novel and specific therapies for ANCA-associated RPGN, and the methods these authors employed to detect this pathway remain relevant for other causes of kidney disease.

\section{Disclosure Statement}

The authors have no conflicts of interest to declare.

\section{Support}

NIH R01 DK084149 (BIF), R01 DK070941 (BIF). 


\section{References}

1 Wang Z, Chen X, Zhang D, Cao Y, Zhang L, Tang W: PYCARD Gene Plays a Key Role in Rapidly Progressive Glomerulonephritis: Results of a Weighted Gene Co-Expression Network Analysis. Am J Nephrol 2018;48:193-204.

2 Langfelder P, Horvath S: WGCNA: an R package for weighted correlation network analysis. BMC Bioinformatics 2008;9:559.

3 Shiohara M, Taniguchi S, Masumoto J, Yasui K, Koike K, Komiyama A, Sagara J: ASC, which is composed of a PYD and a CARD, is up-regulated by inflammation and apoptosis in human neutrophils. Biochem Biophys Res Commun 2002;293:1314-1318.

4 Guo H, Callaway JB, Ting JP: Inflammasomes: mechanism of action, role in disease, and therapeutics. Nat Med 2015;21:677687.

5 Hutton HL, Ooi JD, Holdsworth SR, Kitching AR: The NLRP3 inflammasome in kidney disease and autoimmunity. Nephrology (Carlton) 2016;21:736-744.
6 Ooi JD, Chang J, Hickey MJ, Borza DB, Fugger L, Holdsworth SR, Kitching AR: The immunodominant myeloperoxidase T-cell epitope induces local cell-mediated injury in antimyeloperoxidase glomerulonephritis. Proc Natl Acad Sci U S A 2012;109:E2615-E2624.

7 Schreiber A, Pham CT, Hu Y, Schneider W, Luft FC, Kettritz R: Neutrophil serine proteases promote IL-1 $\beta$ generation and injury in necrotizing crescentic glomerulonephritis. J Am Soc Nephrol 2012;23:470-482. 